\title{
Guest editor's introduction: special issue on analyzing and mining social networks for decision support and recommender systems
}

\author{
I-Hsien Ting ${ }^{1}$
}

Published online: 27 July 2016

(C) Springer Science+Business Media New York 2016

Mining and analyzing social networks is now becoming a very popular research area not only for data mining and web mining but also social network analysis. Data mining is a technique that has the ability to process and analyze large amounts of data and by this to discover valuable information from the data. In recent years, due to the booming of social communications and social network-based web services, data mining has become a very important and powerful technique to process and analyze such large amounts of data.

Recently, many researchers are focusing on developing new data mining techniques and algorithms, or devoting to improve traditional mining techniques for social network analysis. One key test of this discovered data is how it can be used in real-world decision-making situations. Social data are the aggregations of the communications, interactions and experiences of people, and it is useful to leverage this type of data for decision-making. Thus, is an important time to balance the research focus and real world applications, such as decision support and recommender systems.

In this special issue, we invited authors to submit extended versions of their papers, which were deemed top-quality papers from MISNC 2015 (The 2nd Multidisciplinary International Social Networks Conference, Matsuyama, Japan), MSNDS 2015 (The 6th International Workshop on Mining and Analyzing Social Networks for Decision Support, Paris, France) and ASE SocialInformatics 2015 (Kaohsiung, Taiwan). In addition to the invited papers, we also called for public submissions. Each submission was reviewed by at least two experts and revised according to reviewers' comments to ensure the quality of the papers.

The first paper is entitled "Impersonate human decision making process: an interactive context-aware recommender system" and was authored by Chen-Shu Wang, Shiang-Lin Lin and Heng-Li Yang. The authors alter traditional context-aware-recommender systems (CAR) to interactive CAR (iCAR) in order to improve the recommendation accuracy. A car rental

I-Hsien Ting

iting@nuk.edu.tw

1 Department of Information Management, National University of Kaohsiung, No. 700, Kaohsiung

University Road, 811, Kaohsiung City, Taiwan 
website is developed in the paper to demonstrate the concept of iCAR. From the case study, it shows the decision criteria can be clarified by the proposed algorithm for the inference engine of the proposed iCAR.

The second paper is entitled "Social group recommendation in the tourism domain" and was authored by Ingrid Christensen, Silvia Schiaffino and Marcelo G. Armentano. The authors present a social based recommender systems in the domain of tourism, which not only analyzes the preferences of users but also the social relationships between group members. The system combines collaborative, content-based and demographic filtering techniques, which is a hybrid recommender system. It therefore able to generate recommendations to not only an individual but a group of users.

The third paper is entitled "Recommendation using DMF-based fine tuning method" and was authored by Zhiyuan Zhang, Yun Liu, Guandong Xu and Guixun Luo. The authors proposed a so-called Dynamic Matrix Factorization (DMF) which is improved from basic matrix factorization. DMF is a fine tuning method to overcome potential problems in MFbased collaboration filtering recommender systems. In addition, another advantage is that social relations can be easily incorporated into this method and therefore is very useful for social recommendation.

The fourth paper, entitled "RedTweet: recommendation engine for reddit" was authored by Hoang Nguyen, Rachel Richards, Chien-Chun Chan and Kathy J. Liszka. The authors study the use of machine learning (such as SVM and Random Forest) and WordNet-based classifiers to analyze user's tweets to generate an interest profile. The profile is then used to recommend related Reddit threads to the user. A series of experiments were performed to test the performance of machine learning approach and WordNet approach in the paper.

The fifth paper, entitled "Artificial immune network with feature selection for bank term deposit recommendation" was authored by Pei-Chann Chang, Xiao-Yong Lu, Xiao-Qiang Chu, Meng-Hui Chen and Shih-Hsin Chen. In this paper, the authors use Artificial Immune Systems (AIS) as the classification model for collaborative filtering. The authors apply AIS for bank term deposit recommendation to predict the possibility of a new customer to have a term deposit, which is useful for decision making in banking and financial areas.

The sixth paper, entitled "Creating corroborated crisis reports from social media data through formal concept analysis" authored by Simon Andrews, Helen Gibson, Konstantinos Domdouzis and Babak Akhgar. The authors propose a process to extract information related to crisis from social media. The information is near real-time and very useful to assist the decision making of crisis management. In the paper, Nepal 2015 earthquake was used as a case study to demonstrate the proposed process.

The seventh paper, entitled "Inferring social network user profiles using a partial social graph" was authored by Raissa Yapan Dougnon, Philippe Fournier-Viger, Jerry Chun-Wei Lin and Roger Nkambou. The authors proposed the Partial Graph Profile Inference + (PPGPI+) algorithm, which is developed to deal with user profile inference on online social networks. User profile inference are considered as a key for social recommendation as well as for targeted marketing, etc. In the evaluation of the algorithm, it shows the prediction accuracy in some cases achieves $95 \%$ by using PGPI+ for user profile inference.

I-Hsien Ting

Special Issue Guest Editor 